\title{
The current matrix elements from HAL QCD method
}

\author{
Kai Watanabe ${ }^{1, \star}$ and Noriyoshi Ishii ${ }^{1}$ \\ ${ }^{1}$ Research Center for Nuclear physics, Osaka University, Japan
}

\begin{abstract}
HAL QCD method is a method to construct a potential (HAL QCD potential) that reproduces the NN scattering phase shift faithful to the QCD. The HAL QCD potential is obtained from QCD by eliminating the degrees of freedom of quarks and gluons and leaving only two particular hadrons. Therefor, in the effective quantum mechanics of two nucleons defined by HAL QCD potential, the conserved current consists not only of the nucleon current but also an extra current originating from the potential (two-body current). Though the form of the two-body current is closely related to the potential, it is not straight forward to extract the former from the latter. In this work, we derive the the current matrix element formula in the quantum mechanics defined by the HAL QCD potential. As a first step, we focus on the non-relativistic case. To give an explicit example, we consider a second quantized non-relativistic two-channel coupling model which we refer to as the original model. From the original model, the HAL QCD potential for the open channel is constructed by eliminating the closed channel in the elastic two-particle scattering region. The current matrix element formula is derived by demanding the effective quantum mechanics defined by the HAL QCD potential to respond to the external field in the same way as the original two-channel coupling model.
\end{abstract}

\section{Introduction}

Nuclear physics is studied through quantum mechanics of nucleons defined by nucleon-nucleon potentials (NN potentials). In the 90's, several high precision potentials were constructed phenomenologically so as to reproduce the NN scattering data and the deuteron properties[1, 2, 3]. Properties of atomic nuclei such as binding energies, structures and reactions are being studied using these potentials. Recently, a method to construct the NN potential from QCD was proposed by HAL QCD collaboration[4]. In this method, the energy independent non-local NN potential (HAL QCD potential) is constructed by demanding it to reproduce the equal time Nambu-Bethe-Salpeter wave function (NBS wave function) of two nucleons calculated from lattice QCD in the elastic region of the NN scattering. It is shown that the equal time NBS wave functions in the center of mass frame have asymptotic behaviors as $\left.\langle 0|N(\boldsymbol{r}) N(0)| N(\boldsymbol{p}) N(-\boldsymbol{p}), i n\rangle\right|_{r \rightarrow \infty}=Z e^{i \delta(\boldsymbol{p}) \frac{\sin (|\boldsymbol{p} \| \boldsymbol{r}|+\delta(\boldsymbol{p}))}{|\boldsymbol{p}| \boldsymbol{r} \mid}}[5,6,7]$. By definition of the HAL QCD potential, the phase shift is also reproduced by the potential together with the NBS wave function. So, in other words, the HAL QCD method is a procedure to obtain the phase shift equivalent potential from lattice QCD.

In order to calculate the current matrix elements of the nuclei from the quantum mechanics defined by the phase shift equivalent potentials, extra considerations are needed. For example, in

${ }^{\star}$ Speaker, e-mail: kaiw@rcnp.osaka-u.ac.jp 
a system of two-nucleon interacting through OPEP, one may naively consider the proton current $J_{p}^{\mu}(x) \equiv\left(\psi_{p}^{*}(x) \psi_{p}(x),-\frac{\mathrm{i}}{2 m_{p}} \psi_{p}^{*}(x) \overleftrightarrow{\nabla} \psi_{p}(x)\right)$ as the conserved current since proton is the only charged particle that appears explicitly. However, this current does not conserve. In order to define the conserved current, not only the proton current but also the additional contribution originating from the exchanged pion must be taken into account $[8,9]$. In general, since NN interactions consists of exchanges of mesons, there exists additional meson exchange currents(two-body currents) originating from the phase shift equivalent potentials[8]. Thus, the conserved current is a sum of the naive nucleon one-body current $J_{(1)}^{\mu}$ and the two-body current $J_{(2)}^{\mu}$ as $J^{\mu}(x)=J_{(1)}^{\mu}(x)+J_{(2)}^{\mu}(x)$. Here, one may naively expect that the two-body currents have smaller contributions than the one-body currents and to be negligible. However, in some circumstances, two-body currents give dominant contributions to the conserved currents of the nuclear systems. For example, in the $d+\gamma \rightarrow p+n$ reaction, the two-body current of $\mathrm{np}$ system give dominant contribution to the form factor at a specific value of momentum transfer[8]. The determination of the two-body current is a step which can not be skipped in order to consider the conserved current of the atomic nuclei.

The explicit form of the two-body current is closely connected with the explicit form of the potential. However, it is not easy to extract the former from the latter. It can be shown that, the conservation law for the current $J^{\mu}(x)$ constrains the space components of the two-body current $J_{(2)}^{\mu}(x)$ as

$$
\boldsymbol{\nabla} \cdot \boldsymbol{J}_{(2)}(\boldsymbol{x})=-i\left[V, \sum_{i=1,2} e_{i} \delta^{3}\left(\boldsymbol{x}-\boldsymbol{r}_{i}\right)\right]
$$

$[8,10]$. In the equation above, $\mathrm{V}$ denotes the NN potential and $J^{0}(\boldsymbol{x}) \simeq \sum_{i=1,2} e_{i} \delta^{3}\left(\boldsymbol{x}-\boldsymbol{r}_{i}\right)$ is assumed with $\boldsymbol{r}_{i}$ being the position of the $i$-th nucleon. Here, $e_{i}=\frac{1}{2} e\left(I+\tau_{i z}\right)$ stands for the charge where $\tau_{i z}$ is the isospin operator which acts on the $i$-th nucleon. Though the conservation law constrains the two-body currents, it is not strong enough to determine the form uniquely. In the case of OPEP, there exist two currents that satisfy Eq.(1): Sachs current and one pion exchange current (OPEC) [9, 11]. In order to determine the conserved currents, one may think of applying Noether's theorem. However, Noether's theorem can not be applied to theories with potentials such as OPEP, the high precision NN potentials and also the HAL QCD potential. This is because Noether's theorem is not defined in theories with non-local interactions. So, different strategy must be taken. Thompson and Heller derived OPEC by demanding it to reproduce the Bremsstrahlung amplitude calculated from the $\pi \mathrm{N}$ coupling model[11]. Among the Sachs current and the OPEC, latter is believed to be the appropriate one since it reproduces the Bremsstrahlung amplitude while former does not. At any rate, to determine the exchange current, an additional requirement to the current besides of the conservation law is necessary.

In this work, we construct the formula to calculate the current matrix element of the conserved current in the effective quantum mechanics defined by HAL QCD potential. In order to avoid any approximations, as for a first step, we consider a simple but non-trivial Galilei covariant model which we refer to as the original theory: the second quantized non-relativistic two channel coupling model ( $\mathrm{np}-\mathrm{np}^{*}$ coupling model)[12, 13, 14]. Details of this model will be given in section 2. In section 3, the HAL QCD potential is constructed by integrating out the closed channel. The effective quantum mechanics for the open channel in the elastic region is defined. In section 4, the HAL QCD potential in the presence of the external field is defined. In section 5, we derive the current matrix element formula by demanding that effective quantum mechanics defined by the HAL QCD potential responds to the external field in the same way as the original two-channel coupling model. We will give the conclusions and future perspectives in section 6. 


\section{The model}

The model which we employ as the original theory is the second quantized non-relativistic two channel coupling model that is defined by the Hamiltonian $\hat{H} \equiv \hat{T}+\hat{V}$, where $\hat{T} \equiv \hat{T}_{1}+\hat{T}_{2}+\hat{T}_{3}$ denotes the kinetic term where

$$
\begin{aligned}
& \hat{T}_{0} \equiv \int d^{3} x \hat{\phi}_{0}^{\dagger}(\boldsymbol{x})\left(-\frac{\boldsymbol{\nabla}^{2}}{2 m}\right) \hat{\phi}_{0}(\boldsymbol{x}) \\
& \hat{T}_{1} \equiv \int d^{3} x \hat{\phi}_{1}^{\dagger}(\boldsymbol{x})\left(-\frac{\boldsymbol{\nabla}^{2}}{2 m}\right) \hat{\phi}_{1}(\boldsymbol{x}) \\
& \hat{T}_{2} \equiv \int d^{3} x \hat{\phi}_{2}^{\dagger}(\boldsymbol{x})\left(-\frac{\boldsymbol{\nabla}^{2}}{2 m}+\Delta\right) \hat{\phi}_{2}(\boldsymbol{x}) .
\end{aligned}
$$

$\hat{V} \equiv \sum_{\alpha, \beta=1,2} \hat{V}_{\alpha \beta}$ denotes the interaction term

$$
\hat{V}_{\alpha \beta} \equiv \int d^{3} x d^{3} y \hat{\phi}_{0}^{\dagger}(\boldsymbol{x}) \hat{\phi}_{\alpha}^{\dagger}(\boldsymbol{y}) V_{\alpha \beta}(\boldsymbol{x}-\boldsymbol{y}) \hat{\phi}_{\beta}(\boldsymbol{y}) \hat{\phi}_{0}(\boldsymbol{x})
$$

This model mimics the np-np* coupling system[12, 13, 14]. Here, $\hat{\phi}_{0}(\boldsymbol{x}), \hat{\phi}_{1}(\boldsymbol{x})$ and $\hat{\phi}_{2}(\boldsymbol{x})$ correspond to the neutron (n), the proton (p) and an excited proton ( $\left.\mathrm{p}^{*}\right)$ whose excitation energy is $\Delta$, respectively. In this paper, we use fonts such as $\boldsymbol{x}$ and $\boldsymbol{y}$ to indicate three dimensional vectors and $\hat{*}$ to indicate that they are operators acting on the Fock space. Also, we will treat all the particles as elementary bosons. Thus the operators corresponding to the particles satisfy the commutation relation $\left[\hat{\phi}_{\alpha}(\boldsymbol{x}), \hat{\phi}_{\beta}^{\dagger}(\boldsymbol{y})\right]=$ $\delta_{\alpha \beta} \delta^{3}(x-y)$, and all the other combinations vanish. The eigenvalue relation for $\hat{H}$ is $\hat{H}|n, \boldsymbol{P}\rangle=$ $E_{n}\left(\boldsymbol{P}^{2}\right)|n, \boldsymbol{P}\rangle$, where $\boldsymbol{P}$ is the total momentum and index n labels the intrinsic excitation in the center of mass frame. $E_{n}\left(\boldsymbol{P}^{2}\right)$ and $|n, \boldsymbol{P}\rangle$ are the energy eigenvalue and the state respectively. These states are normalized as $\langle m, \boldsymbol{Q} \mid n, \boldsymbol{P}\rangle=(2 \pi)^{3} \delta_{m n} \delta^{3}(\boldsymbol{P}-\boldsymbol{Q})$. Using the Galilei covariance, the energy eigenvalue is decomposed into two parts $E_{n}\left(\boldsymbol{P}^{2}\right)=\widetilde{E}_{n}+\frac{1}{4 m} \boldsymbol{P}^{2}$ where $\widetilde{E}_{n}$ denotes the intrinsic energy.

The model has a U(1) symmetry generated by the charge $\hat{Q}_{\mathrm{p}} \equiv \int d^{3} x\left(\hat{\phi}_{1}^{\dagger}(\boldsymbol{x}) \hat{\phi}_{1}(\boldsymbol{x})+\hat{\phi}_{2}^{\dagger}(\boldsymbol{x}) \hat{\phi}_{2}(\boldsymbol{x})\right)$ which is equivalent to the sum of the number of proton and its excited state. The U(1) current operator in this model is defined as

$$
\hat{j}^{\mu}(x) \equiv \sum_{j=1,2}\left[\delta_{0}^{\mu} \hat{\phi}_{j}^{\dagger}(x) \hat{\phi}_{j}(x)-\sum_{k=1,2,3} \delta_{k}^{\mu} \frac{\mathrm{i}}{2 m} \hat{\phi}_{j}^{\dagger}(x) \overleftrightarrow{\partial_{k}} \hat{\phi}_{j}(x)\right]
$$

Here, $k$ and $\overleftrightarrow{\partial}$ indicates the spatial coordinates and the differential operator that acts on fields coming to its left and right sides as $\overleftarrow{\partial}-\vec{\partial}$ respectively. $\hat{\phi}_{\alpha}(x) \equiv \hat{e}^{i \hat{H} x_{0}} \phi_{\alpha}(\boldsymbol{x}) e^{-i \hat{H} x_{0}}$ denotes Heisenberg field operator.

In this work, we will focus on the two particle subspace $\mathcal{F}$ which is spanned by the states as $|\psi\rangle \equiv \int d^{3} x d^{3} y\left(\phi_{0}^{\dagger}(\boldsymbol{x}) \phi_{1}^{\dagger}(\boldsymbol{y})|0\rangle \psi_{1}(\boldsymbol{x}, \boldsymbol{y})+\phi_{0}^{\dagger}(\boldsymbol{x}) \phi_{2}^{\dagger}(\boldsymbol{y})|0\rangle \psi_{2}(\boldsymbol{x}, \boldsymbol{y})\right)$, where $|0\rangle$ denotes the nonrelativistic vacuum. Also, we introduce a cutoff by using a projection operator $\hat{\mathbb{P}}_{\Lambda} \equiv|0\rangle\langle 0|+$ $\sum_{n}^{\widetilde{E}_{n}<\Lambda} \int \frac{d^{3} P}{(2 \pi)^{3}}|n, \boldsymbol{P}\rangle\langle n, \boldsymbol{P}|$ and the truncated subspace $\mathcal{F}_{\Delta}$ is defined by $\mathcal{F}_{\Delta} \equiv \hat{\mathbb{P}}_{\Delta} \cdot \mathcal{F}$. We will use this projection to construct the HAL QCD potential in the external field in section 4.

Using the field operators and the energy eigenstates, the equal time Nambu-Bethe-Salpeter wave functions (NBS wave function) for the $\mathrm{np}(i=1)$ and $\mathrm{np}^{*}(i=2)$ channels are defined as,

$$
\psi_{i}(\boldsymbol{x}, \boldsymbol{y} ; t \mid n, \boldsymbol{P}) \equiv\left\langle 0\left|\hat{\phi}_{0}(\boldsymbol{x}, t) \hat{\phi}_{i}(\boldsymbol{y}, t)\right| n, \boldsymbol{P}\right\rangle
$$


By making use of Galilei covariance, NBS wave function can be factorized as

$$
\psi_{\alpha}(\boldsymbol{x}, \boldsymbol{y}, t \mid n, \boldsymbol{P})=\widetilde{\psi}_{\alpha}(\boldsymbol{r}, t \mid n) \exp (i \boldsymbol{P} \cdot \boldsymbol{R}) \exp \left(-i \frac{1}{4 m} \boldsymbol{P}^{2} t\right)
$$

where $\boldsymbol{R}$ is the center of mass coordinate and $\boldsymbol{r}=\boldsymbol{y}-\boldsymbol{x}$ is the relative coordinate with $\boldsymbol{x}$ and $\boldsymbol{y}$ being the position of $\mathrm{n}$ and $\mathrm{p}\left(\mathrm{p}^{*}\right) . \widetilde{\psi}_{\alpha}(\boldsymbol{r}, t \mid n)$ denotes a reduced NBS wave function defined as

$$
\widetilde{\psi}_{\alpha}(\boldsymbol{r}, t \mid n) \equiv\left\langle 0\left|\hat{\phi}_{0}(\boldsymbol{r} / 2, t) \hat{\phi}_{\alpha}(-\boldsymbol{r} / 2, t)\right| n, \boldsymbol{P}=\mathbf{0}\right\rangle \text {. }
$$

The reduced NBS wave function satisfies the coupled channel Schödinger equation

$$
\begin{gathered}
\left(\widetilde{E}_{n}+\frac{\nabla_{r}^{2}}{2 \widetilde{m}}\right) \widetilde{\psi}_{1}(\boldsymbol{r} \mid n)=V_{11}(\boldsymbol{r}) \widetilde{\psi}_{1}(\boldsymbol{r} \mid n)+V_{12}(\boldsymbol{r}) \widetilde{\psi}_{2}(\boldsymbol{r} \mid n) \\
\left(\widetilde{E}_{n}+\frac{\nabla_{\boldsymbol{r}}^{2}}{2 \widetilde{m}}-\Delta\right) \widetilde{\psi}_{2}(\boldsymbol{r} \mid n)=V_{21}(\boldsymbol{r}) \widetilde{\psi}_{1}(\boldsymbol{r} \mid n)+V_{22}(\boldsymbol{r}) \widetilde{\psi}_{2}(\boldsymbol{r} \mid n),
\end{gathered}
$$

where $\widetilde{m} \equiv m / 2$ denotes the reduced mass.

\section{HAL QCD potential and the effective quantum mechanics}

We will apply HAL QCD method to our model to obtain an effective np potential which is used to construct the effective quantum mechanics for the np channel. In order to obtain the reduced HAL QCD potential $\widetilde{\mathcal{V}}\left(\boldsymbol{r}, \boldsymbol{r}^{\prime}\right)$, we firstly demand the reduced NBS wave functions of the states with energies of no more than $\Delta$ to satisfy the Schrödinger equation

$$
\left(\widetilde{E}_{n}+\frac{1}{2 \widetilde{m}} \nabla_{\boldsymbol{r}}^{2}\right) \widetilde{\psi}_{1}(\boldsymbol{r} \mid n)=\int d^{3} \boldsymbol{r}^{\prime} \widetilde{\mathcal{V}}\left(\boldsymbol{r}, \boldsymbol{r}^{\prime}\right) \widetilde{\psi}_{1}\left(\boldsymbol{r}^{\prime} \mid n\right) .
$$

This Schrödinger equation is satisfied by the following form of the non-local energy independent potential

$$
\widetilde{\mathcal{V}}\left(\boldsymbol{r}, \boldsymbol{r}^{\prime}\right) \equiv \sum_{m}^{\widetilde{E}_{m}<\Delta}\left(V_{11}(\boldsymbol{r}) \widetilde{\psi}_{1}(\boldsymbol{r} \mid m)+V_{12}(\boldsymbol{r}) \widetilde{\psi}_{2}(\boldsymbol{r} \mid m)\right) \widetilde{\psi}_{1}^{\vee}\left(\boldsymbol{r}^{\prime} \mid m\right)
$$

Here, we introduced the dual vector $\widetilde{\psi}_{1}^{\vee}(\boldsymbol{r} \mid m)$ which is constructed as $\widetilde{\psi}_{1}^{\vee}(\boldsymbol{r} \mid n) \equiv \sum_{m}^{\widetilde{E}_{m}<\Delta}\left(\widetilde{\mathcal{N}}^{-1}\right)_{n m} \widetilde{\psi}_{1}^{*}(\boldsymbol{r} \mid m)$, with the norm kernel $\widetilde{\mathcal{N}}_{n m} \equiv \int d^{3} r \widetilde{\psi}_{1}^{*}(\boldsymbol{r} \mid n) \widetilde{\psi}_{1}(\boldsymbol{r} \mid m)$. The dual vector and the reduced NBS wave function satisfy the orthogonality relation by definition as $\int d^{3} r \widetilde{\psi}_{1}^{\vee}(\boldsymbol{r} \mid m) \widetilde{\psi}_{1}(\boldsymbol{r} \mid n)=\delta_{m n}$. Using these expressions, it is straight forward to prove that the right hand side of eq(10) is equivalent to that of eq( 8$)$ for states with energy eigenvalues no more than $\Delta$. By making use of the Galilei covariance, it is possible to generalize the reduced HAL QCD potential to the potential for arbitrary frame (not only the center of mass frame) $\mathcal{V}\left(\boldsymbol{x}, \boldsymbol{y} ; \boldsymbol{x}^{\prime}, \boldsymbol{y}^{\prime}\right)$. Here, we will show only the results.

$$
\mathcal{V}\left(\boldsymbol{x}, \boldsymbol{y} ; \boldsymbol{x}^{\prime}, \boldsymbol{y}^{\prime}\right) \equiv \widetilde{\mathcal{V}}\left(\boldsymbol{x}-\boldsymbol{y} ; \boldsymbol{x}^{\prime}-\boldsymbol{y}^{\prime}\right) \delta^{3}\left(\frac{\boldsymbol{x}+\boldsymbol{y}}{2}-\frac{\boldsymbol{x}^{\prime}+\boldsymbol{y}^{\prime}}{2}\right) .
$$

The Schrödinger equation is then generalized to

$$
\left(E_{n}\left(\boldsymbol{P}^{2}\right)+\frac{1}{2 m} \nabla_{\boldsymbol{x}}^{2}+\frac{1}{2 m} \nabla_{y}^{2}\right) \psi_{1}(\boldsymbol{x}, \boldsymbol{y} \mid n, \boldsymbol{P})=\int d^{3} x^{\prime} d^{3} y^{\prime} \mathcal{V}\left(\boldsymbol{x}, \boldsymbol{y} ; \boldsymbol{x}^{\prime}, \boldsymbol{y}^{\prime}\right) \psi_{1}\left(\boldsymbol{x}^{\prime}, \boldsymbol{y}^{\prime} \mid n, \boldsymbol{P}\right)
$$


Farther details of the derivations are given in [14].

For later discussions, we will briefly introduce the eigenvalue property of the effective quantum mechanics. Firstly, for the center of mass frame, we have the eigenvalue equations as

$$
\begin{aligned}
& \widetilde{\mathcal{E}}_{n} \widetilde{\chi}_{n}^{\mathrm{R}}(\boldsymbol{r})=-\frac{\nabla^{2}}{2 \widetilde{m}} \widetilde{\chi}_{n}^{\mathrm{R}}(\boldsymbol{r})+\int d^{3} r^{\prime} \widetilde{\mathcal{V}}\left(\boldsymbol{r}, \boldsymbol{r}^{\prime}\right) \widetilde{\chi}_{n}^{\mathrm{R}}\left(\boldsymbol{r}^{\prime}\right), \\
& \widetilde{\mathcal{E}}_{n} \widetilde{\chi}_{n}^{\mathrm{L}}(\boldsymbol{r})=-\frac{\nabla^{2}}{2 \widetilde{m}} \widetilde{\chi}_{n}^{\mathrm{L}}(\boldsymbol{r})+\int d^{3} r^{\prime} \widetilde{\chi}_{n}^{\mathrm{L}}\left(\boldsymbol{r}^{\prime}\right) \widetilde{\mathcal{V}}\left(\boldsymbol{r}^{\prime}, \boldsymbol{r}\right) .
\end{aligned}
$$

where $\widetilde{\mathcal{E}}_{n}$ is the energy eigenvalue. The HAL QCD potential is defined so as to reproduce the NBS wave functions in the elastic region of $n p$ scattering. This implies that, for the states with reduced energy eigenvalues less than $\Delta$, the reduced right eigenvector $\widetilde{\chi}_{n}^{\mathrm{R}}(\boldsymbol{r})$ is equal to the reduced NBS wave function. Therefor, we have the relation $\widetilde{\chi}_{n}^{\mathrm{R}}(\boldsymbol{r})=\widetilde{\psi}_{1}(\boldsymbol{r} \mid n)$ for $\widetilde{\mathcal{E}}_{n}=\widetilde{E}_{n} \quad\left(\widetilde{\mathcal{E}}_{n}<\Delta\right)$.

Using Galilei covariance, the reduced eigenvectors are generalized to arbitrary frames as $\chi_{n, \boldsymbol{P}}^{\mathrm{R}, \mathrm{L}}(\boldsymbol{x}, \boldsymbol{y}) \equiv \widetilde{\chi}_{n}^{\mathrm{R}}(\boldsymbol{x}-\boldsymbol{y}) \cdot \exp \left( \pm i \boldsymbol{P} \cdot \frac{1}{2}(\boldsymbol{x}+\boldsymbol{y})\right)$ where $\chi_{n}^{\mathrm{L}}(\boldsymbol{x}, \boldsymbol{y})$ and $\chi_{n}^{\mathrm{R}}(\boldsymbol{x}, \boldsymbol{y})$ are the right and left eigenvectors. The sign in the exponential is plus for the right and minus for the left eigenvectors respectively. Associated with the energy eigenvalue $\mathcal{E}_{n}\left(\boldsymbol{P}^{2}\right) \equiv \overline{\mathcal{E}}_{n}+\frac{1}{4 m} \boldsymbol{P}^{2}$, the eigenvalue equation for these vectors are given as

$$
\begin{aligned}
& \mathcal{E}_{n}\left(\boldsymbol{P}^{2}\right) \chi_{n, \boldsymbol{P}}^{\mathrm{R}}(\boldsymbol{x}, \boldsymbol{y})=\left(-\frac{\boldsymbol{\partial}_{\boldsymbol{x}}^{2}}{2 m}-\frac{\boldsymbol{\partial}_{\boldsymbol{y}}^{2}}{2 m}\right) \chi_{n, \boldsymbol{P}}^{\mathrm{R}}(\boldsymbol{x}, \boldsymbol{y})+\int d^{3} x^{\prime} d^{3} y^{\prime} \mathcal{V}\left(\boldsymbol{x}, \boldsymbol{y} ; \boldsymbol{x}^{\prime}, \boldsymbol{y}^{\prime}\right) \chi_{n, \boldsymbol{P}}^{\mathrm{R}}\left(\boldsymbol{x}^{\prime}, \boldsymbol{y}^{\prime}\right) \\
& \mathcal{E}_{n}\left(\boldsymbol{P}^{2}\right) \chi_{n, \boldsymbol{P}}^{\mathrm{L}}(\boldsymbol{x}, \boldsymbol{y})=\left(-\frac{\boldsymbol{\partial}_{\boldsymbol{x}}^{2}}{2 m}-\frac{\boldsymbol{\partial}_{\boldsymbol{y}}^{2}}{2 m}\right) \chi_{n, \boldsymbol{P}}^{\mathrm{L}}(\boldsymbol{x}, \boldsymbol{y})+\int d^{3} x^{\prime} d^{3} y^{\prime} \chi_{n, \boldsymbol{P}}^{\mathrm{L}}\left(\boldsymbol{x}^{\prime}, \boldsymbol{y}^{\prime}\right) \mathcal{V}\left(\boldsymbol{x}^{\prime}, \boldsymbol{y}^{\prime} ; \boldsymbol{x}, \boldsymbol{y}\right) .
\end{aligned}
$$

These left and right eigenvectors satisfy the orthogonal relations[14]. Here, the right eigenvector is equal to the NBS wave function of the $n p$ channel in the elastic region.

\section{HAL QCD potential in the external field}

In order to calculate the matrix element of the U(1) current in the effective quantum mechanics which was introduced in the previous section, we will use the external field method. In this work, we will consider a space-time dependent external field $A_{\mu}(\boldsymbol{x}, t)$. The current matrix elements are derived by demanding the response of the effective quantum mechanics to the external field to be same as the response of the original theory. To do so, we firstly introduce the external field to the original theory. Regarding the fact that the conserved U(1) current is as given in eq.(4), the external field couples to the kinetic term of the Hamiltonian as $\hat{H}\left[A_{t}\right] \equiv \hat{T}\left[A_{t}\right]+\hat{V}$. Here, the kinetic terms are given as

$$
\begin{aligned}
\hat{T}_{0} & \equiv \int d^{3} x \hat{\phi}_{0}^{\dagger}(\boldsymbol{x})\left(-\frac{\boldsymbol{\partial}^{2}}{2 m}\right) \hat{\phi}_{0}(\boldsymbol{x}) \\
\hat{T}_{1}\left[A_{t}\right] & \equiv \int d^{3} x \hat{\phi}_{1}^{\dagger}(\boldsymbol{x})\left(-\frac{(\boldsymbol{\partial}-i \boldsymbol{A}(\boldsymbol{x}, t))^{2}}{2 m}-A_{0}(\boldsymbol{x}, t)\right) \hat{\phi}_{1}(\boldsymbol{x}) \\
\hat{T}_{2}\left[A_{t}\right] & \equiv \int d^{3} x \hat{\phi}_{2}^{\dagger}(\boldsymbol{x})\left(-\frac{(\boldsymbol{\partial}-i \boldsymbol{A}(\boldsymbol{x}, t))^{2}}{2 m}-A_{0}(\boldsymbol{x}, t)+\Delta\right) \hat{\phi}_{2}(\boldsymbol{x}) .
\end{aligned}
$$

Because the external field has a time-dependence, it shifts the states in the subspace $\mathcal{F}_{\Delta}$ to the region above the $\mathrm{np}^{*}$ threshold. Consequently, it triggers unwanted transitions harmful when constructing the low-energy effective quantum mechanics. In order to construct the effective quantum 
mechanics, we need to terminate these transitions. This can be achieved by generating the time evolution by the truncated Hamiltonian $\hat{H}_{\Delta}\left[A_{t}\right] \equiv \hat{\mathbb{P}}_{\Delta} \hat{H}\left[A_{t}\right] \hat{\mathbb{P}}_{\Delta}$. The time evolution operator which we will refer to as the truncated time evolution operator is expressed as the time ordered product of this truncated Hamiltonian as

$$
\hat{U}_{\Delta}(t, s ; A) \equiv \sum_{n=0}^{\infty}(-i)^{n} \int_{s}^{t} d t_{n} \int_{s}^{t_{n}} d t_{n-1} \cdots \int_{s}^{t_{2}} d t_{1} \hat{H}_{\Delta}\left[A_{t_{n}}\right] \hat{H}_{\Delta}\left[A_{t_{n-1}}\right] \cdots \hat{H}_{\Delta}\left[A_{t_{1}}\right] .
$$

In the external field, the equal time NBS wave function with the truncated time evolution operator is defined as

$$
\psi_{\alpha}^{(\Delta)}(\boldsymbol{x}, \boldsymbol{y}, t ; A \mid n, \boldsymbol{P}) \equiv\left\langle 0\left|\hat{\phi}_{0}^{(\Delta)}(\boldsymbol{x}, t ; A) \hat{\phi}_{i}^{(\Delta)}(\boldsymbol{y}, t ; A)\right| n, \boldsymbol{P}\right\rangle
$$

for $i=1,2$. Here, $\hat{\phi}_{\alpha}^{(\Delta)}(\boldsymbol{y}, t ; A) \equiv \hat{U}_{\Delta}(0, t ; A) \hat{\phi}_{\alpha}(\boldsymbol{x}) \hat{U}_{\Delta}(t, 0 ; A)$ denotes the Heisenberg operator for the particles evolving in time by the truncated operator. Since the introduction of the external field breaks the Galilei covariance, this truncated NBS wave function can not be reduced any more unlike eq.(6). The truncated NBS wave function satisfy the coupled channel Schrödinger equation in the external field

$$
\begin{aligned}
& \left(i \partial_{t}+\frac{\boldsymbol{\partial}_{\boldsymbol{x}}^{2}}{2 m}+\frac{\boldsymbol{D}_{\boldsymbol{y}}^{2}}{2 m}+A_{0}(\boldsymbol{y}, t)\right) \psi_{1}^{(\Delta)}(\boldsymbol{x}, \boldsymbol{y}, t ; A \mid n, \boldsymbol{P}) \\
& =\int d^{3} x^{\prime} d^{3} y^{\prime} \\
& \left.\quad \times \quad V_{11}\left(\boldsymbol{x}, \boldsymbol{y} ; \boldsymbol{x}^{\prime}, \boldsymbol{y}^{\prime} ; A_{t}\right) \psi_{1}^{(\Delta)}(\boldsymbol{x}, \boldsymbol{y}, t ; A \mid n, \boldsymbol{P})+V_{12}\left(\boldsymbol{x}, \boldsymbol{y} ; \boldsymbol{x}^{\prime}, \boldsymbol{y}^{\prime} ; A_{t}\right) \psi_{2}^{(\Delta)}(\boldsymbol{x}, \boldsymbol{y}, t ; A \mid n, \boldsymbol{P})\right\} \\
& \left(i \partial_{t}+\frac{\boldsymbol{\partial}_{\boldsymbol{x}}^{2}}{2 m}+\frac{\boldsymbol{D}_{\boldsymbol{y}}^{2}}{2 m}+A_{0}(\boldsymbol{y}, t)-\Delta\right) \psi_{2}^{(\Delta)}(\boldsymbol{x}, \boldsymbol{y}, t ; A \mid n, \boldsymbol{P}) \\
& \quad=\int d^{3} x^{\prime} d^{3} y^{\prime} \\
& \left.\quad \times \quad V_{21}\left(\boldsymbol{x}, \boldsymbol{y} ; \boldsymbol{x}^{\prime}, \boldsymbol{y}^{\prime} ; A_{t}\right) \psi_{1}^{(\Delta)}(\boldsymbol{x}, \boldsymbol{y}, t ; A \mid n, \boldsymbol{P})+V_{22}\left(\boldsymbol{x}, \boldsymbol{y} ; \boldsymbol{x}^{\prime}, \boldsymbol{y}^{\prime} ; A_{t}\right) \psi_{2}^{(\Delta)}(\boldsymbol{x}, \boldsymbol{y}, t ; A \mid n, \boldsymbol{P})\right\} .
\end{aligned}
$$

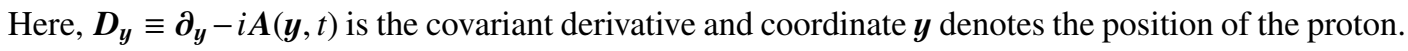
The coupled channel potentials $V_{i j}\left(\boldsymbol{x}, \boldsymbol{y} ; \boldsymbol{x}^{\prime}, \boldsymbol{y}^{\prime} ; A_{t}\right)(i, j=1,2)$ has been modified by the existence of the external field and the cutoff and its exact form is

$$
V_{\alpha \beta}\left(\boldsymbol{x}, \boldsymbol{y} ; \boldsymbol{x}^{\prime}, \boldsymbol{y}^{\prime} ; A_{t}\right) \equiv V_{\alpha \beta}(\boldsymbol{x}-\boldsymbol{y}) \delta^{3}\left(\boldsymbol{x}-\boldsymbol{x}^{\prime}\right) \delta^{3}\left(\boldsymbol{y}-\boldsymbol{y}^{\prime}\right)+\Delta V_{\alpha \beta}\left(\boldsymbol{x}, \boldsymbol{y} ; \boldsymbol{x}^{\prime}, \boldsymbol{y}^{\prime} ; A_{t}\right),
$$

with

$$
\Delta V_{\alpha \beta}\left(\boldsymbol{x}, \boldsymbol{y} ; \boldsymbol{x}^{\prime}, \boldsymbol{y}^{\prime} ; A_{t}\right) \equiv-\left\langle 0\left|\hat{\phi}_{0}(\boldsymbol{x}) \hat{\phi}_{\alpha}(\boldsymbol{y})\left(\hat{\mathbb{I}}-\hat{\mathbb{P}}_{\Delta}\right) \hat{H}\left[A_{t}\right] \hat{\mathbb{P}}_{\Delta} \hat{\phi}_{0}^{\dagger}\left(\boldsymbol{x}^{\prime}\right) \hat{\phi}_{\beta}^{\dagger}\left(\boldsymbol{y}^{\prime}\right)\right| 0\right\rangle .
$$

The additional term $\Delta V_{\alpha \beta}(\cdots)$ originates from the projection operator and terminates the additional shifts by the external field. If it were not for $A_{\mu}(\boldsymbol{x}, t)$, the additional term vanishes due to the factor $\left(\hat{\mathbb{I}}-\hat{\mathbb{P}}_{\Delta}\right) \hat{H}\left[A_{t}\right] \hat{\mathbb{P}}_{\Delta}$. Also note that, the term has time dependence since it depends on the external field.

The effective Schrödinger equation and the HAL QCD potential in the external field can be defined from the truncated NBS wave function. The result is

$$
\begin{aligned}
& \left(i \partial_{t}+\frac{\boldsymbol{\partial}_{\boldsymbol{x}}^{2}}{2 m}+\frac{\boldsymbol{D}_{\boldsymbol{y}}^{2}}{2 m}+A_{0}(\boldsymbol{y}, t)\right) \psi_{1}^{(\Delta)}(\boldsymbol{x}, \boldsymbol{y}, t ; A \mid n, \boldsymbol{P}) \\
& =\int d^{3} x^{\prime} d^{3} y^{\prime} \mathcal{V}\left(\boldsymbol{x}, \boldsymbol{y} ; \boldsymbol{x}^{\prime}, \boldsymbol{y}^{\prime} ; A_{t}\right) \psi_{1}^{(\Delta)}\left(\boldsymbol{x}^{\prime}, \boldsymbol{y}^{\prime}, t ; A \mid n, \boldsymbol{P}\right),
\end{aligned}
$$


where,

$$
\begin{aligned}
\mathcal{V}\left(\boldsymbol{x}, \boldsymbol{y} ; \boldsymbol{x}^{\prime}, \boldsymbol{y}^{\prime} ; A_{t}\right) & \\
\equiv & \sum_{m=0}^{\widetilde{E}_{m}<\Delta} \int d^{3} x^{\prime \prime} d^{3} y^{\prime \prime} \\
& \left\{V_{11}\left(\boldsymbol{x}, \boldsymbol{y} ; \boldsymbol{x}^{\prime \prime}, \boldsymbol{y}^{\prime \prime} ; A_{t}\right) \widetilde{\psi}_{1}\left(\boldsymbol{x}^{\prime \prime}-\boldsymbol{y}^{\prime \prime} \mid m\right)+V_{12}\left(\boldsymbol{x}, \boldsymbol{y} ; \boldsymbol{x}^{\prime \prime}, \boldsymbol{y}^{\prime \prime} ; A_{t}\right) \widetilde{\psi}_{2}\left(\boldsymbol{x}^{\prime \prime}-\boldsymbol{y}^{\prime \prime} \mid m\right)\right\} \\
& \times \widetilde{\psi}_{1}^{\vee}\left(\boldsymbol{x}^{\prime}-\boldsymbol{y}^{\prime} \mid m\right) \delta^{3}\left(\frac{\boldsymbol{x}^{\prime \prime}+\boldsymbol{y}^{\prime \prime}}{2}-\frac{\boldsymbol{x}^{\prime}+\boldsymbol{y}^{\prime}}{2}\right) .
\end{aligned}
$$

\section{The current matrix element formula}

After defining the effective quantum mechanics and deriving its Schrödinger equation, the current matrix element for the open np channel can be calculated in a closed way. By demanding the response of the effective quantum mechanics to the external field to be equal to that of the original theory, we obtain the current matrix element formula in the effective quantum mechanics. For any states $|m, \boldsymbol{Q}\rangle$, $|n, \boldsymbol{P}\rangle \in \mathcal{F}_{\Delta}$, the matrix element of the current operator $\hat{j}^{\mu}(\boldsymbol{z})$ is given in the form

$$
\begin{aligned}
& \left\langle m, \boldsymbol{Q}\left|\hat{j}^{\mu}(\boldsymbol{z})\right| n, \boldsymbol{P}\right\rangle \\
& \quad=\int d^{3} x d^{3} y \int d^{3} x^{\prime} d^{3} y^{\prime} \chi_{m, \boldsymbol{Q}}^{\mathrm{L}}(\boldsymbol{x}, \boldsymbol{y}) K^{\mu}\left(\boldsymbol{x}, \boldsymbol{y} ; \boldsymbol{x}^{\prime}, \boldsymbol{y}^{\prime} ; \boldsymbol{z}\right) \chi_{n, \boldsymbol{P}}^{\mathrm{R}}\left(\boldsymbol{x}^{\prime}, \boldsymbol{y}^{\prime}\right) .
\end{aligned}
$$

Here, $K^{\mu}\left(\boldsymbol{x}, \boldsymbol{y} ; \boldsymbol{x}^{\prime}, \boldsymbol{y}^{\prime} ; \boldsymbol{z}\right)$ is the current operator in the effective quantum mechanics

$$
\begin{aligned}
& K^{0}\left(\boldsymbol{x}, \boldsymbol{y} ; \boldsymbol{x}^{\prime}, \boldsymbol{y}^{\prime} ; \boldsymbol{z}\right) \delta\left(t-z_{0}\right) \\
& \quad \equiv-\delta^{3}(\boldsymbol{z}-\boldsymbol{y}) \delta^{3}\left(\boldsymbol{x}-\boldsymbol{x}^{\prime}\right) \delta^{3}\left(\boldsymbol{y}-\boldsymbol{y}^{\prime}\right) \delta\left(t-z_{0}\right)+\left.\frac{\delta \mathcal{V}\left(\boldsymbol{x}, \boldsymbol{y} ; \boldsymbol{x}^{\prime}, \boldsymbol{y}^{\prime} ; A_{t}\right)}{\delta A_{0}\left(\boldsymbol{z}, z_{0}\right)}\right|_{A \equiv 0} \\
& K^{i}\left(\boldsymbol{x}, \boldsymbol{y} ; \boldsymbol{x}^{\prime}, \boldsymbol{y}^{\prime} ; z\right) \delta\left(t-z_{0}\right) \\
& \quad \equiv \quad \overleftrightarrow{\boldsymbol{\partial}}_{z}^{i} \delta^{3}(z-\boldsymbol{y}) \delta^{3}\left(\boldsymbol{x}-\boldsymbol{x}^{\prime}\right) \delta^{3}\left(\boldsymbol{y}-\boldsymbol{y}^{\prime}\right) \delta\left(t-z_{0}\right)+\left.\frac{\delta \mathcal{V}\left(\boldsymbol{x}, \boldsymbol{y} ; \boldsymbol{x}^{\prime}, \boldsymbol{y}^{\prime} ; A_{t}\right)}{\delta A_{i}\left(z, z_{0}\right)}\right|_{A \equiv 0}
\end{aligned}
$$

The detail of the derivation is in our latest paper [14]. Here, we give some comments on this formula.

1. In the conventional quantum mechanics defined by the hermitian Hamiltonian, the current matrix elements are derived by sandwiching the current operator with the state vector and its hermitian conjugate. However, since the HAL QCD potential is non-hermitian, the matrix element is derived by sandwiching the operator with the left and right eigenvectors.

2. The first term of the current operator $K^{i}\left(\boldsymbol{x}, \boldsymbol{y} ; \boldsymbol{x}^{\prime}, \boldsymbol{y}^{\prime} ; \boldsymbol{z}\right) \delta\left(t-z_{0}\right)$ represents the naive one-body current. The second term represents the two-body current.

3. In a realistic circumstances, the HAL QCD potentials are some times defined using derivative expansion. However, the same strategy as given here works even if the construction procedure of the HAL QCD potential is different from the one demonstrated in section 3 and 4. 


\section{Conclusion}

In this work, we have derived the formula to calculate the current matrix element in the effective quantum mechanics defined by the HAL QCD potential. The current matrix element obtained here is faithful to the original non-relativistic model. In order to give an explicit example, we employed the non-relativistic $\mathrm{np}-\mathrm{np}^{*}$ two channel coupling model as the original model so as to avoid any approximations. From the model, the effective quantum mechanics for the open np channel was constructed by eliminating the closed np* channel by applying the HAL QCD method. The effective np potential (HAL QCD potential) was obtained by demanding it to reproduce the equal time NBS wave function in the elastic scattering region for the np channel. We also constructed the HAL QCD potential in the presence of the external gauge field. By demanding the effective quantum mechanics to have the same response to the external field as the original theory, the current matrix element formula was determined in a closed analytic form.

To use our result in lattice QCD simulations, farther considerations are necessary. Firstly, we need to generalize our method to relativistic framework in order to apply it to QCD. Secondly, we have to deal with the composite particles such as hadrons. In other words, we need to take into account the form factors which we did not consider at present. Finally, we have to deal with the time-evolution in the presence of the external field with the cutoff. In order to achieve our goals, some approximations may be necessary.

\section{Acknowledgements}

We thank Prof. M. Oka and Prof. W. Bentz and Dr. M. Yamanaka for fruitful discussions. This work was supported by JSPS KAKENHI Grands No. JP25400244, RCNP theory group and by the Japanese Ministry of Education, Culture, Sports, Science and Technology as "Priority Issue on Post-K computer" (Elucidation of the Fundamental Laws and Evolution of the Universe) and Joint Institute for Computational Fundamental Science.

\section{References}

[1] R. B. Wiringa, V. G. J. Stoks and R. Schiavilla, Phys. Rev. C 51, 38 (1995)

[2] V. G. J. Stoks, R. A. M. Klomp, C. P. F. Terheggen and J. J. de Swart, Phys. Rev. C 49 (1994) 2950

[3] R. Machleidt, Phys. Rev. C 63 (2001) 024001

[4] N. Ishii, S. Aoki and T. Hatsuda, Phys. Rev. Lett. 99 (2007) 022001

[5] C. J. D. Lin, G. Martinelli, C. T. Sachrajda and M. Testa, Nucl. Phys. B 619 (2001) 467

[6] S. Aoki et al. [CP-PACS Collaboration], Phys. Rev. D 71 (2005) 094504

[7] S. Aoki, T. Hatsuda and N. Ishii, Prog. Theor. Phys. 123 (2010) 89

[8] D. O. Riska, Phys. Rept. 181 (1989) 207.

[9] R. G. Sachs, Phys. Rev. 74 (1948) 433.

[10] K. Ohta, Phys. Rev. C 39 (1989) 2302.

[11] R. H. Thompson and L. Heller, Phys. Rev. C 7 (1973) 2355.

[12] M. C. Birse, arXiv:1208.4807 [nucl-th].

[13] T. Sugiura, N. Ishii and M. Oka, Phys. Rev. D 95 (2017) no.7, 074514

[14] K. Watanabe and N. Ishii arXiv:1709.09424 [hep-lat] 